(C) American Chemical Society, Chem. Mater., cm052717x, Supporting Info Page S1

\title{
Periodic mesoporous organosilicas with ethane and large isocyanurate bridging groups
}

\section{Rafal M. Grudzien, ${ }^{a}$ Bogna E. Grabicka, ${ }^{a}$ Stanislaw Pikus ${ }^{b}$ and Mietek Jaroniec ${ }^{a} *$}

${ }^{a}$ Department of Chemistry, Kent State University, Kent, OH 44242, USA. Fax: +1 330672

3816; Tel: +1 330672 3790; E-mail:jaroniec@kent.edu

${ }^{b}$ Department of Crystallography, M. Curie-Skłodowska University, 20-031 Lublin, Poland

Table 1S. Parameters obtained from Small Angle X-ray Scattering (SAXS) according to the P6mm symmetry group for the extracted PMOs studied.

\begin{tabular}{|c|c|c|c|c|c|c|c|}
\hline \multirow{2}{*}{$\underset{\delta^{a}}{\text { Sample }}$} & \multicolumn{2}{|c|}{ Synthesis mixture } & \multirow[b]{2}{*}{$2 \theta(\text { deg. })^{b}$} & \multirow[b]{2}{*}{$d(\mathrm{~nm})^{\mathrm{c}}$} & \multirow[b]{2}{*}{$I / I_{0}^{d}$} & \multirow[b]{2}{*}{$h k l^{e}$} & \multirow[b]{2}{*}{$\mathrm{a}(\mathrm{nm})^{f}$} \\
\hline & $\begin{array}{l}n_{B T S E} \\
\text { (mmole) }\end{array}$ & $\begin{array}{l}n_{I C S} \\
\text { (mmole) }\end{array}$ & & & & & \\
\hline \multirow{5}{*}{$\begin{array}{l}\text { EI-0 } \\
0.078\end{array}$} & \multirow{5}{*}{5.164} & \multirow{5}{*}{0} & 0.790 & 11.17 & 1000 & 100 & 12.90 \\
\hline & & & 1.360 & 6.48 & 11 & 110 & 12.97 \\
\hline & & & 1.570 & 5.62 & 16 & 200 & 12.98 \\
\hline & & & 2.086 & 4.23 & 2 & 210 & 12.94 \\
\hline & & & 2.346 & 3.77 & 1 & 300 & 13.05 \\
\hline \multirow[b]{2}{*}{ EI-a } & \multirow{4}{*}{5.035} & \multirow{4}{*}{0.086} & 0.830 & 10.64 & 1000 & 100 & 12.29 \\
\hline & & & & & & 110 & \\
\hline \multirow[t]{2}{*}{0.092} & & & 1.658 & 5.32 & 11 & 200 & 12.30 \\
\hline & & & 2.204 & 4.01 & 2 & 210 & 12.25 \\
\hline \multirow{5}{*}{ EI-b } & \multirow{5}{*}{4.906} & \multirow{5}{*}{0.172} & 0.815 & 10.84 & 1000 & 100 & 12.52 \\
\hline & & & 1.403 & 6.29 & 1 & 110 & 12.59 \\
\hline & & & 1.630 & 5.42 & 9 & 200 & 12.78 \\
\hline & & & 2.154 & 4.10 & 2 & 210 & 12.53 \\
\hline & & & 2.813 & 3.14 & 1 & 220 & 12.56 \\
\hline \multirow[b]{2}{*}{ EI-1 } & \multirow{4}{*}{4.647} & \multirow{4}{*}{0.344} & 0.790 & 11.17 & 1000 & 100 & 12.90 \\
\hline & & & 1.370 & 6.45 & 2 & 110 & 12.90 \\
\hline \multirow{2}{*}{0.086} & & & 1.580 & 5.59 & 11 & 200 & 12.92 \\
\hline & & & 2.093 & 4.22 & 1 & 210 & 12.90 \\
\hline \multirow[b]{2}{*}{ EI-2 } & \multirow{4}{*}{4.131} & \multirow{4}{*}{0.688} & 0.820 & 10.75 & 1000 & 100 & 12.41 \\
\hline & & & & & & 110 & \\
\hline \multirow[t]{2}{*}{0.118} & & & 1.630 & 5.42 & 20 & 200 & 12.52 \\
\hline & & & 2.148 & 4.11 & 11 & 210 & 12.56 \\
\hline \multirow{4}{*}{0.101} & \multirow{4}{*}{3.615} & \multirow{4}{*}{1.038} & 0.800 & 11.07 & 1000 & 100 & 12.78 \\
\hline & & & & & & 110 & \\
\hline & & & 1.580 & 5.57 & 6 & 200 & 12.86 \\
\hline & & & 2.107 & 4.19 & 2 & 210 & 12.81 \\
\hline
\end{tabular}

${ }^{a} \delta$ - Full width at half maximum of the most intense peak; ${ }^{b} 2 \theta$ - scattering angle; ${ }^{c} d$ - interplanar spacing; ${ }^{d} I / I_{0}-$ relative intensity; ${ }^{e} h k l$ - Muller indexes; ${ }^{f} \mathrm{a}$ - unit cell parameter. 


\section{(C) American Chemical Society, Chem. Mater., cm052717x, Supporting Info Page S2}

Table 2S. Parameters obtained from Small Angle X-ray Scattering (SAXS) according to the I-cubic symmetry group for the extracted PMOs studied.

\begin{tabular}{|c|c|c|c|c|c|c|c|}
\hline \multirow{2}{*}{ Sample } & \multicolumn{2}{|c|}{ Synthesis mixture } & \multirow[b]{2}{*}{$2 \theta(\mathrm{deg} .)^{b}$} & \multirow[b]{2}{*}{$d(\mathrm{~nm})^{\mathrm{c}}$} & \multirow[b]{2}{*}{$\mathrm{I} / \mathrm{I}_{0}{ }^{d}$} & \multirow[b]{2}{*}{$h k l^{e}$} & \multirow[b]{2}{*}{$\mathrm{a}(\mathrm{nm})^{f}$} \\
\hline & $\begin{array}{l}n_{B T S E} \\
\text { (mmole) }\end{array}$ & $\begin{array}{l}n_{I C S} \\
\text { (mmole) }\end{array}$ & & & & & \\
\hline \multirow{3}{*}{$\begin{array}{c}\text { EI-2 } \\
0.118\end{array}$} & \multirow{3}{*}{4.131} & \multirow{3}{*}{0.688} & 0.820 & 10.75 & 1000 & 110 & 15.20 \\
\hline & & & 1.630 & 5.42 & 20 & 220 & 15.33 \\
\hline & & & 2.148 & 4.11 & 11 & 321 & 15.38 \\
\hline \multirow[b]{3}{*}{0.101} & \multirow{3}{*}{3.615} & \multirow{3}{*}{1.038} & 0.800 & 11.07 & 1000 & 110 & 15.65 \\
\hline & & & 1.580 & 5.57 & 6 & 220 & 15.70 \\
\hline & & & 2.107 & 4.19 & 2 & 321 & 15.68 \\
\hline
\end{tabular}

${ }^{a} \delta$ - Full width at half maximum of the most intense peak; ${ }^{b} 2 \theta$ - scattering angle; ${ }^{c} d$ - interplanar spacing; ${ }^{d} I / I_{0}-$ relative intensity; ${ }^{e} h k l$ - Muller indexes; ${ }^{f_{a}}$ - unit cell parameter.
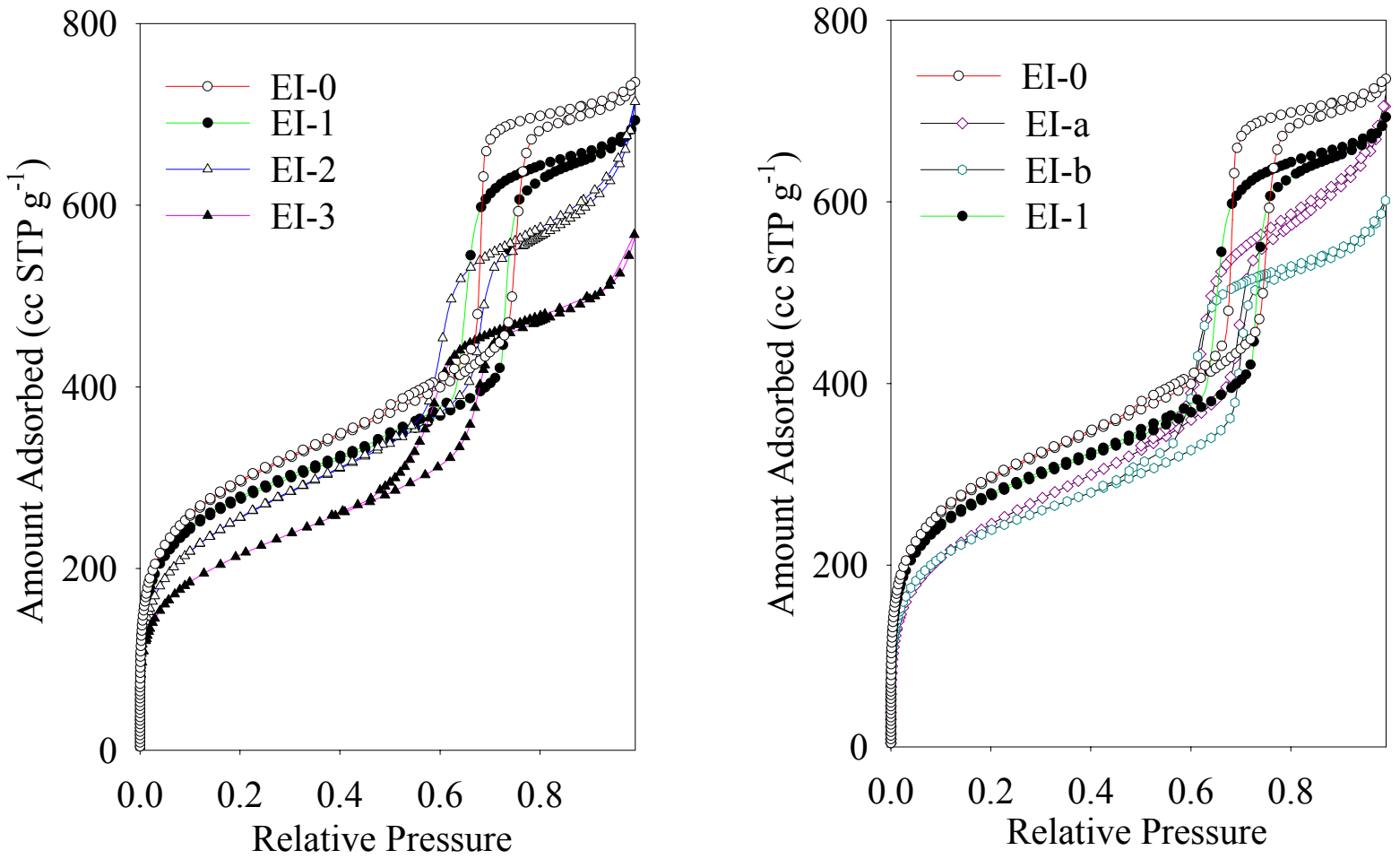

Figure 1S. Comparison of nitrogen adsorption-desorption isotherms at $-196 \mathrm{C}$ for a series of the extracted PMOs studied (a) and for the extracted PMOs with additonal EI-a and EI-b samples (b) 
(C) American Chemical Society, Chem. Mater., cm052717x, Supporting Info Page S3

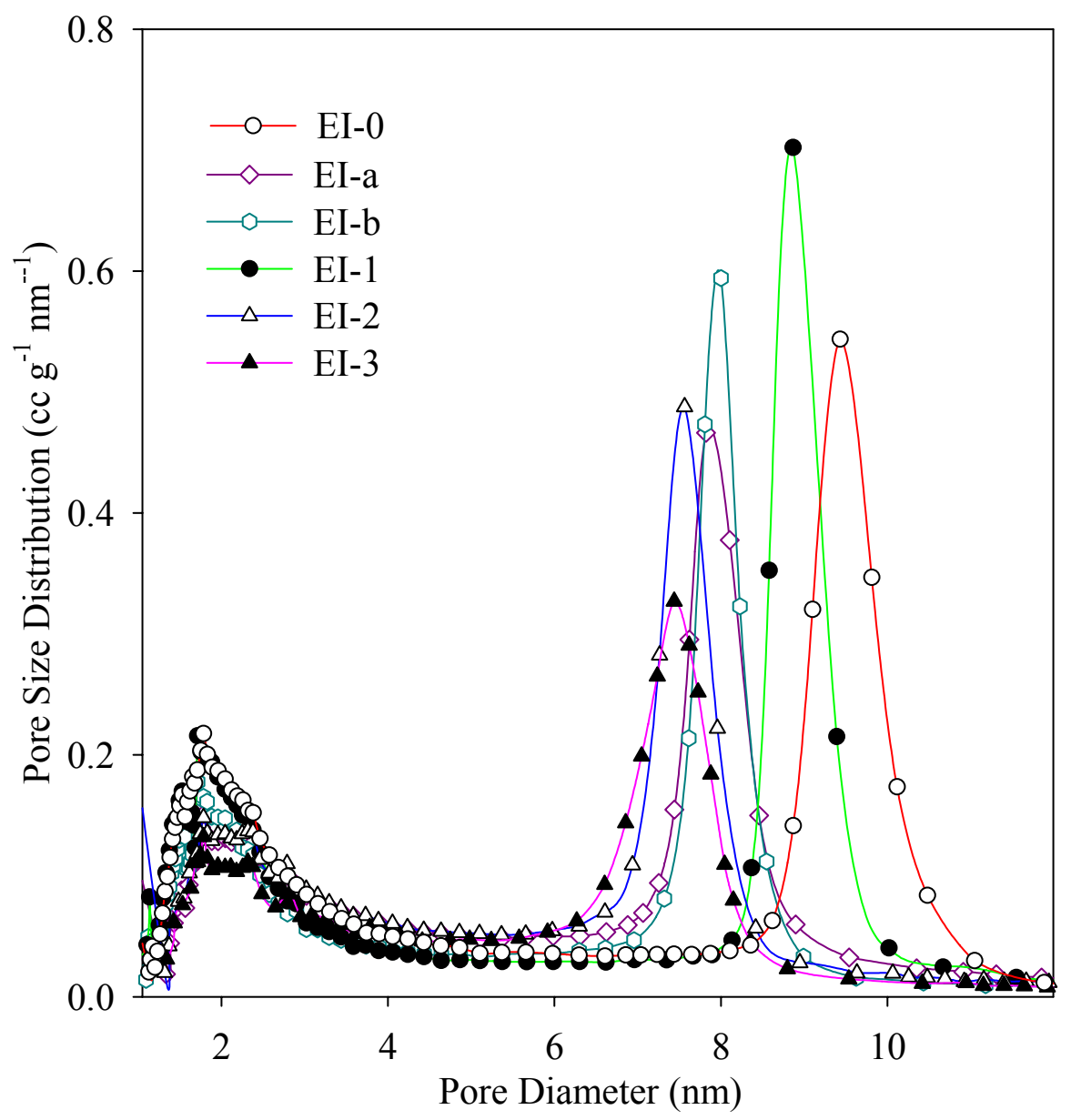

Figure 2S. Comparison of the pore size distributions (PSDs) calculated according to the KJS method for the PMOs studied.

Table 3S. Adsorption properties of the EI-a and EI-b PMO samples determined from nitrogen adsorption data. ${ }^{\mathrm{a}}$

\begin{tabular}{|c|c|c|c|c|c|}
\hline Sample & $\begin{array}{c}S_{\mathrm{BET}} \\
\mathbf{m}^{2} \mathbf{g}^{-1}\end{array}$ & $\begin{array}{c}V_{m} \\
C g^{-1}\end{array}$ & $\begin{array}{c}V_{p} \\
c^{-1} g^{-1}\end{array}$ & $\begin{array}{c}V_{t} \\
\text { cc g }^{-1}\end{array}$ & $\begin{array}{c}\mathbf{W}_{\mathrm{KJS}} \\
\mathbf{n m}\end{array}$ \\
\hline EI-a & 895 & 0.25 & 0.64 & 1.10 & 7.8 \\
\hline EI-b & 856 & 0.25 & 0.57 & 0.94 & 8.0 \\
\hline
\end{tabular}

${ }^{a} \mathrm{~S}_{\mathrm{BET}}$, BET specific surface area; $\mathrm{V}_{\mathrm{m}}$, volume of pores below $4 \mathrm{~nm} ; \mathrm{V}_{\mathrm{p}}$, volume of primary pores calculated between 4 and $12 \mathrm{~nm} ; \mathrm{V}_{\mathrm{t}}$, single-point pore volume; $\mathrm{w}_{\mathrm{KJS}}$, mesopore diameter. 


\section{(C) American Chemical Society, Chem. Mater., cm052717x, Supporting Info Page S4}

Table 4S. Adsorption properties of the EI-2 PMO sample before and after thermal degradation of isocyanurate bridging group determined from nitrogen adsorption data.

\begin{tabular}{|c|c|c|c|c|c|c|}
\hline Sample & $\begin{array}{c}\mathrm{S}_{\mathrm{BET}} \\
\mathbf{m}^{2} \mathbf{g}^{-1}\end{array}$ & $\begin{array}{c}V_{m} \\
\text { cc }^{-1}\end{array}$ & $\underset{\text { cc } \mathrm{g}^{-1}}{\mathrm{~V}_{\mathrm{C}}}$ & $\begin{array}{c}V_{t} \\
\text { cc } g^{-1}\end{array}$ & $\begin{array}{c}\mathbf{W}_{\mathrm{KJS}} \\
\mathbf{n m}\end{array}$ & $\underset{\mathbf{n m}}{\mathbf{d}}$ \\
\hline EI-2 & 933 & 0.27 & 0.61 & 1.05 & 7.56 & 10.90 \\
\hline EI-2-c ${ }^{*}$ & 673 & 0.16 & 0.34 & 0.64 & 5.60 & 7.81 \\
\hline
\end{tabular}

${ }^{a} \mathrm{~S}_{\mathrm{BET}}$, BET specific surface area; $\mathrm{V}_{\mathrm{m}}$, volume of pores below $4 \mathrm{~nm} ; \mathrm{V}_{\mathrm{p}}$, volume of primary pores calculated between 4 and $12 \mathrm{~nm} ; \mathrm{V}_{\mathrm{t}}$, single-point pore volume; $\mathrm{w}_{\mathrm{KJS}}$, mesopore diameter; $\mathrm{d}$, interplanar spacing obtained for the first peak; * extracted EI-2 sample was calcined in flowing air at $550{ }^{\circ} \mathrm{C}$ for 3 hours

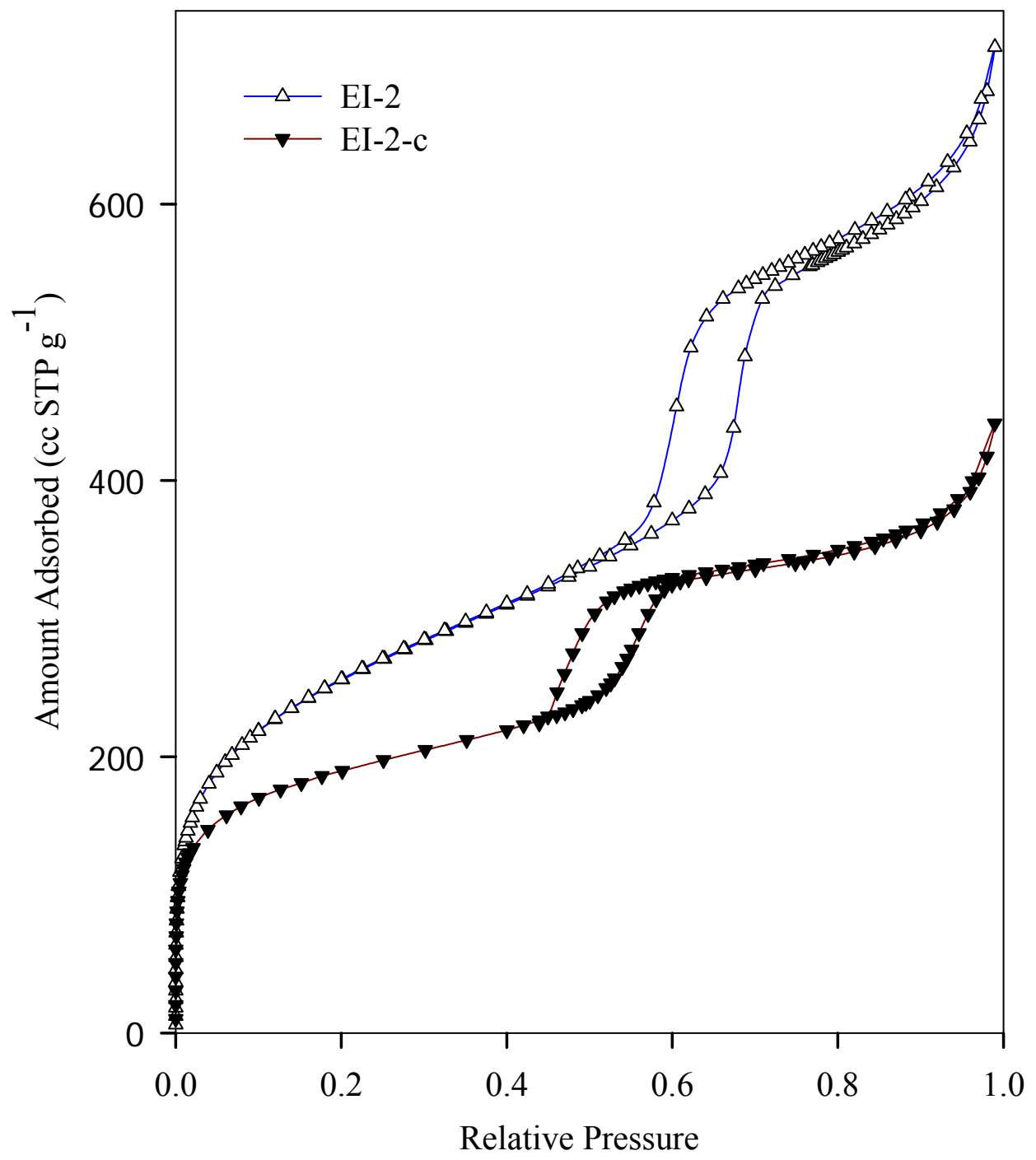

Figure 3S. Comparison of nitrogen adsorption-desorption isotherms at $-196 \mathrm{C}$ for extracted (EI-2) PMO sample and the same sample subjected to calcination at $550{ }^{\circ} \mathrm{C}$ in flowing air for 3 hours. 
(C) American Chemical Society, Chem. Mater., cm052717x, Supporting Info Page S5

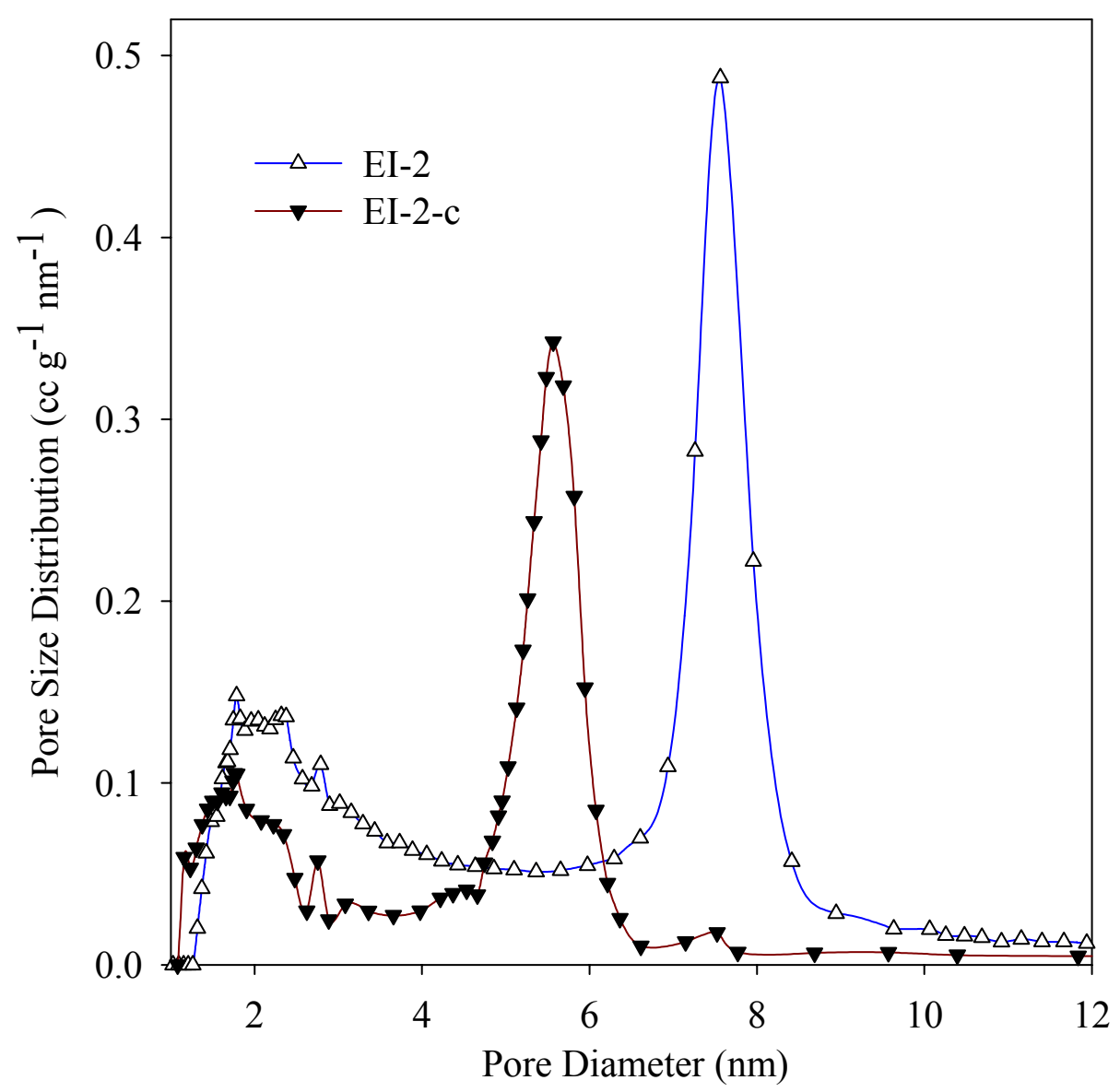

Figure 4S. Comparison of the pore size distributions (PSDs) calculated according to the KJS method for extracted (EI-2) PMO sample and the same sample subjected to calcination at $550{ }^{\circ} \mathrm{C}$ in flowing air for 3 hours. 
(C) American Chemical Society, Chem. Mater., cm052717x, Supporting Info Page S6

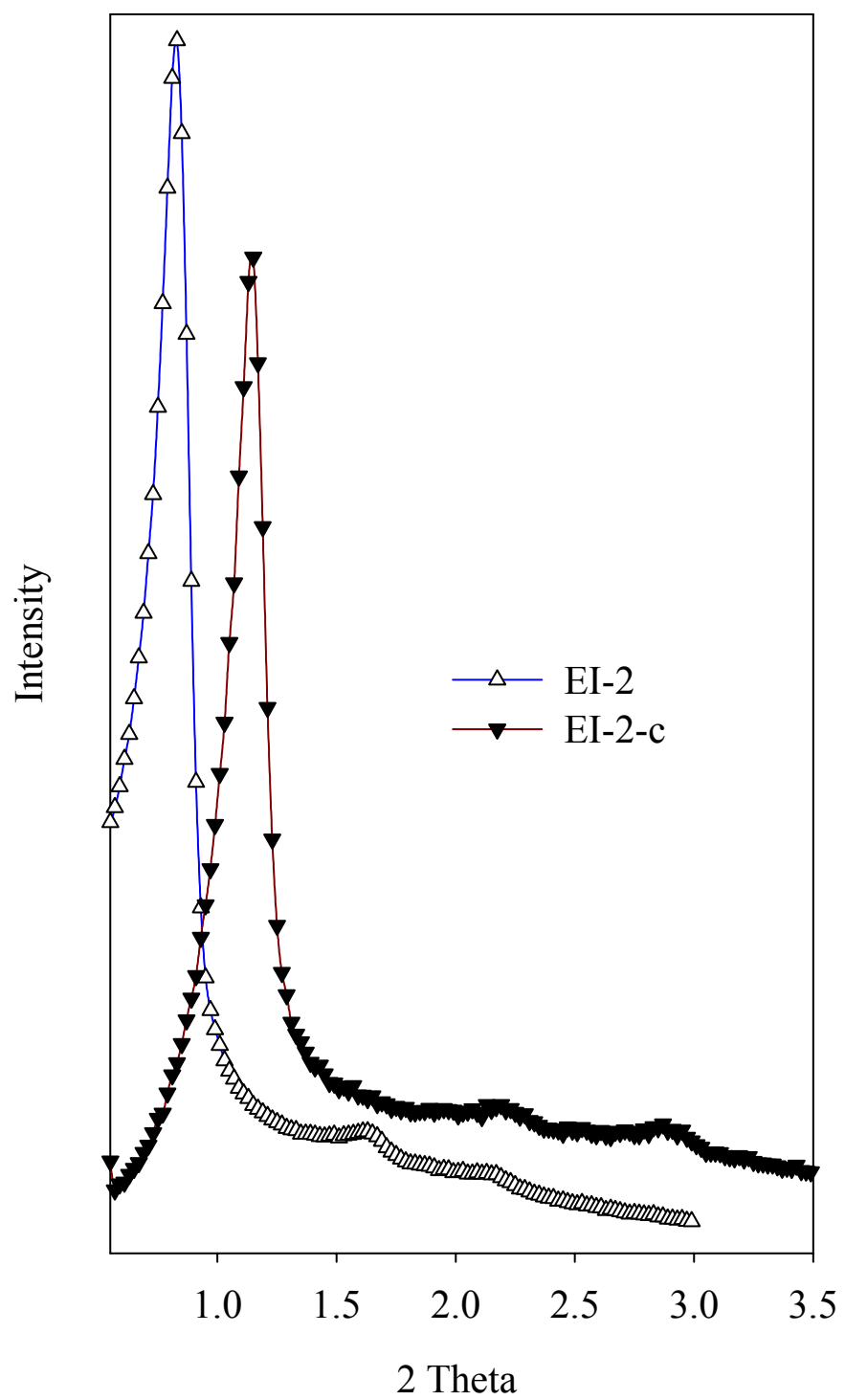

Figure 5S. Comparison of the X-Ray diffraction patterns for extracted (EI-2) PMO sample and the same sample subjected to calcination at $550^{\circ} \mathrm{C}$ in flowing air for 3 hours. The XRD patterns were recorded using a PANanalytical, Inc. $\mathrm{X}^{\prime}$ Pert Pro (MPD) Multi Purpose Diffractometer with $\mathrm{Cu} \mathrm{K \alpha}$ radiation, operating voltage of $40 \mathrm{kV}, 0.02^{\circ}$ step size and $20 \mathrm{~s}$ step time at room temperature. 